\title{
Fiber Reinforced Cartilage ECM Functionalized Bioinks for Functional Cartilage Tissue Engineering
}

\author{
Swetha Rathan ${ }^{1,2}$, Léa Dejob ${ }^{1,3}$, Rossana Schipani ${ }^{1,2}$, Benjamin Haffner ${ }^{4}$, \\ Matthias E. Möbius ${ }^{4}$, Daniel J. Kelly $y^{1,2,5,6^{*}}$ \\ ${ }^{1}$ Trinity Centre for Bioengineering, Trinity Biomedical Sciences Institute, \\ Trinity College Dublin, Ireland.
}

${ }^{2}$ Department of Mechanical and Manufacturing Engineering, School of Engineering, Trinity College Dublin, Ireland.

${ }^{3}$ Ecole Nationale Supérieure de Chimie de Mulhouse, Université de Haute-Alsace, Mulhouse, France.

${ }^{4}$ School of Physics, Trinity College Dublin, Ireland.

${ }^{5}$ Advanced Materials and Bioengineering Research Centre (AMBER), Royal College of Surgeons in Ireland and Trinity College Dublin, Ireland.

${ }^{6}$ Department of Anatomy, Royal College of Surgeons in Ireland, Dublin, Ireland.

*Corresponding Author: Tel: +353-1-8963947, email: kellyd9@tcd.ie.

Keywords: Extracellular matrix bioinks; 3D-Bioprinting; Polycaprolactone; Articular cartilage tissue engineering; Growth factor 


\begin{abstract}
Focal articular cartilage (AC) defects, if left untreated, can lead to debilitating diseases such as osteoarthritis. While several tissue engineering strategies have been developed to promote cartilage regeneration, it is still challenging to generate functional $A C$ capable of sustaining high load-bearing environments. We developed a new class of cartilage extracellular matrix (cECM)-functionalized alginate bioink for the bioprinting of cartilaginous tissues. The bioinks were 3D-printable, supported mesenchymal stem cell (MSC) viability post-printing and robust chondrogenesis in vitro, with the highest levels of COLLII and ACAN expression observed in bioinks containing the highest concentration of cECM. Enhanced chondrogenesis in cECM-functionalized bioinks was also associated with progression along an endochondral-like pathway, as evident by increases in RUNX2 expression and calcium deposition in vitro. The bioinks loaded with MSCs and TGF-ß3 were also found capable of supporting robust chondrogenesis, opening the possibility of using such bioinks for direct 'print-and-implant' cartilage repair strategies. Finally, we demonstrated that networks of 3D-printed polycaprolactone fibers with compressive modulus comparable to native $A C$ could be used to mechanically reinforce these bioinks, with no loss in cell viability. It is envisioned that combinations of such biomaterials can be used in multiple-tool biofabrication strategies for the bioprinting of biomimetic cartilaginous implants.
\end{abstract}




\section{Introduction}

Focal articular cartilage $(\mathrm{AC})$ damage can occur due to age, ${ }^{[1]}$ trauma, ${ }^{[2]}$ repetitive injury, ${ }^{[3]}$ joint misalignment, ${ }^{[4]}$ osteochondritis, ${ }^{[5]}$ and osteonecrosis ${ }^{[6-7]}$ and is very common, especially in the young and active population. ${ }^{[8]} \mathrm{AC}$ is avascular, which contributes to the limited regenerative capacity of the tissue. ${ }^{[9]}$ If such cartilage defects are left untreated, they typically progress, which can predispose patients to osteoarthritis. ${ }^{[10]} \mathrm{A}$ number of surgical strategies have been developed to repair cartilage defects including autologous chondrocyte implantation $(\mathrm{ACl})$, micro-fracture and autologous and allogenic osteochondral grafting. ${ }^{[11]}$ Although these repair techniques can relieve pain and lead to some improvement in function, the repaired cartilage is typically more fibrocartilaginous in nature and not capable of withstanding the intense mechanical environment of the joint in the long-term. ${ }^{[12]}$ Other major drawbacks include a paucity of donors (in the case of allogenic osteochondral grafting), graft failure, inflammation, donor site morbidity, and de-differentiation of injected chondrocytes in the case of ACl. ${ }^{[6]}$

Over the past 25 years, numerous tissue engineering strategies have emerged for regenerating AC. ${ }^{[13]}$ Mesenchymal stem cells (MSCs) are also widely being used as an alternative to chondrocytes for cartilage tissue engineering. ${ }^{[14-17]}$ To successfully engineer cartilage-like tissue using MSCs, it is critical to develop biomaterials that (i) provide a microenvironment conducive to a stable chondrogenic phenotype, (ii) provide mechanical integrity, and (iii) possess the capacity to deliver growth factors and other regulatory biomolecules. In an effort to engineer cartilage-mimetic biomaterials favorable to chondrogenesis, a number of studies have investigated the chondro-inductivity of natural polymer scaffolds generated from and/or functionalized with commercially available collagens ${ }^{[18-25]}$ (typically type I or type II), hyaluronic acid, ${ }^{[19,26-28]}$ chondroitin sulphate ${ }^{[29-30]}$ and decellularized cartilage extracellular matrix (cECM). ${ }^{[25,31-38]}$ While such ECM derived biomaterials are generally supportive of a chondrogenic phenotypic, they typically lack the mechanical properties for functioning in high load bearing environments. 
3D-Bioprinting has emerged as a promising strategy to engineer mechanically functional implants for orthopaedic tissue engineering. ${ }^{[22,39-45]} \mathrm{An}$ advantage of bioprinting is that soft, cell-friendly bioinks can be mechanically reinforced with networks of stiffer polymers such as polycaprolactone $(P C L)^{[39-40,45-46]}$ to engineer composites that are both mechanically functional and supportive of new tissue deposition by resident cells. PCL is widely used in 3D-bioprinting for its mechanical strength, biocompatibility, low melting temperatures as well as high thermal stability and can be used as a supporting material to regenerate a variety of tissues. ${ }^{[46-48]}$ Perhaps a bigger challenge is the identification of bioinks that are highly printable and supportive of a chondrogenic phenotype. Bioinks derived from natural polymers such as gelatin ${ }^{[49-50]}$ and alginate ${ }^{[22,40,42-43,51]}$ are commonly used in 3D-bioprinting as they are biocompatible, printable and easily modifiable to tune their stiffness and rate of degradation; however, they are not believed to provide specific cues supportive of chondrogenesis. In contrast, bioinks derived from decellularized cECM have been shown to be highly supportive of chondrogenesis, ${ }^{[31,52]}$ however, they are more difficult to modify than other natural polymers.

An alternative but attractive approach would be to functionalize a routinely used hydrogel bioink such as alginate with $\mathrm{CECM}$. Alginate is a naturally derived polysaccharide and a versatile biomaterial[53-55] owing to its excellent biocompatibility, gelation properties, tuneable stiffness, ${ }^{[43,56]}$ injectability, ${ }^{[53]}$ printability ${ }^{[57]}$ and ability to support the differentiation of encapsulated cells. ${ }^{[51,58]}$ Although widely used for cartilage tissue engineering applications, alginate is often modified, eg., by immobilization of cell adhesion ligands such as arginine-glycine-asparagine (RGD) sequences for enhanced cell attachment ${ }^{[59]}$ or combined with other naturally derived biomaterials such as chitosan, ${ }^{[60]}$ hyaluronic acid, ${ }^{[26]}$ or collagen ${ }^{[22]}$ for improved chondrogenesis.

The objectives of this study were to 1) develop 3D-printable cECM-functionalized alginate bioinks and assess their chondrogenic potential in vitro; 2) evaluate the capacity of these cECM-functionalized bioinks to deliver chondrogenic growth factors (specifically TGF- 33 ); and 3) develop a 3D-printing strategy to fabricate a composite, biomimetic implant consisting of biological (cECM-functionalized 
bioink containing MSCs) and mechanical (PCL framework) components. To address these objectives, alginate was first functionalized with two different concentrations $(0.2 \%$ and $0.4 \%(\mathrm{w} / \mathrm{v}))$ of cECM and the rheology and 3D-printability of the resultant bioinks were assessed. Next, the chondro-inductive potential of these bioinks as well as their capacity to act as a TGF- $\beta 3$ delivery system was investigated. Then, 3D-printing parameters were optimized to fabricate a porous PCL framework with articular cartilage-like stiffness. Finally, a hybrid construct was fabricated using a 3D-printed PCL network containing a cECM-functionalized bioink and evaluated for its overall mechanical properties and biocompatibility.

\section{Materials and Methods}

Isolation, expansion and culture conditions of human or porcine bone marrow MSCs.

Human bone marrow-derived MSCs (hBMSCs) were purchased from Lonza (Switzerland) and tripotentiality was confirmed before use. Porcine bone marrow-derived MSCs (pBMSCs) were isolated from the femora of 3-4 month old pigs (>50 kg) within $3 \mathrm{~h}$ of sacrifice and expanded. ${ }^{[61]} \mathrm{pBMSCs}$ or hBMSCs were plated at a seeding density of $5 \times 10^{3} \mathrm{cells} / \mathrm{cm}^{2}$ in standard culture media, corresponding to Dulbecco's modified Eagle's Medium (DMEM) supplemented with GlutaMAX (Gibco, Ireland), 10\% fetal bovine serum (FBS, Gibco, Ireland), 100 units $/ \mathrm{mL}$ penicillin, $100 \mu \mathrm{g} / \mathrm{mL}$ streptomycin (both Gibco, Ireland) and $5 \mathrm{ng} / \mathrm{mL}$ fibroblast growth factor-2 (FGF-2; ProSpec, Israel) at $37^{\circ} \mathrm{C}$ in a humidified atmosphere at $37{ }^{\circ} \mathrm{C}, 5 \% \mathrm{CO}_{2}$, and $20 \% \mathrm{O}_{2}$ with medium change every $2-3$ days. ${ }^{[58]}$ When passaged, cells were plated at $4.6 \times 10^{3}$ cells $/ \mathrm{cm}^{2}$ ( 0.8 million cells per T-175 flask). For chondrogenic differentiation of the MSCs, cells were expanded until passage 3 (pBMSCs) or 4 (hBMSCs), trypsinized and resuspended in basic chemically defined media (CDM) containing DMEM+ GlutaMAX ${ }^{\mathrm{TM}}$ (Gibco, Ireland), $1 \mathrm{mM}$ sodium pyruvate, $350 \mu \mathrm{M}$ L-proline, $1.5 \mathrm{mg} / \mathrm{mL}$ bovine serum albumin (all from Sigma-Aldrich, Ireland), 100 units $/ \mathrm{mL}$ penicillin and $100 \mu \mathrm{g} / \mathrm{mL}$ streptomycin (both Gibco, Ireland). ${ }^{58]}$ To prepare fully supplemented chondrogenic media (FCDM) for chondrogenic differentiation of the MSCs, basic CDM media was supplemented with $1 \mathrm{X}$ insulin transferrin-selenium, $100 \mathrm{nM}$ dexamethasone, $300 \mu \mathrm{M}$ 
ascorbic acid, and $17 \mu \mathrm{M}$ linolenic acid (all from Sigma Aldrich, Ireland) and TGF- $\beta 3$ (10 ng/mL, PrepoTech, UK).

\section{Extraction of cartilage-derived extracellular matrix (CECM)}

To extract the cECM, knee joints of 2-3 months old pigs were obtained from a local butcher and AC was aseptically harvested. Following a previously established protocol, ${ }^{[44,62]} \mathrm{CECM}$ was extracted from $\mathrm{AC}$ under sterile conditions. Briefly, the AC was finely diced into 1 to $3 \mathrm{~mm}$ pieces using a scalpel and subsequently solubilized in activated pepsin solution (1500 Units/mg of wet cartilage). A type II collagen enriched $\mathrm{CECM}$ was then selectively extracted by salt precipitation using $0.9 \mathrm{M} \mathrm{NaCl}$. The resultant precipitate was re-suspended in $0.5 \mathrm{M}$ acetic acid solution and transferred to a Spectra Por dialysis membrane (MWCO 10KDa, Cole-Parmer, UK). The dialysis was carried out against $0.02 \mathrm{M} \mathrm{Na}_{2} \mathrm{HPO}_{4}$ (PH 9.4) for 24 hours at $4^{\circ} \mathrm{C}$. The dialyzed $\mathrm{CECM}$ was then freeze-dried and stored at $-80^{\circ} \mathrm{C}$. Stock solution of $20 \mathrm{mg} / \mathrm{mL} \mathrm{cECM}$ was prepared using $0.5 \mathrm{~N} \mathrm{CH}_{3} \mathrm{COOH}$ and neutralized to $\mathrm{pH} 7.4$ using $0.2 \mathrm{~N}$ $\mathrm{NaCl}$. All the chemicals were obtained from Sigma-Aldrich, Ireland.

\section{Formulation of cECM-functionalized alginate bioinks}

Using a dual syringe approach, stock solution of cECM $(20 \mathrm{mg} / \mathrm{mL})$ was first mixed with $\mathrm{hBMSCs}$ or pBMSCs (final concentration of cells $20 \times 10^{6} / \mathrm{mL}$ ) and subsequently mixed with $4.9 \%(\mathrm{w} / \mathrm{v}$ ) sodium alginate (LVG, NovaMatrix, Norway) solution in CDM to prepare two cECM-functionalized alginate bioinks: 1) alginate $(2.45 \%)+0.2 \%(w / v) c E C M ; 2)$ alginate $(2.45 \%)+0.4 \%(w / v) c E C M$. Alginate $(2.45 \%)$ without CECM was used as a control. Bioinks used for 3D-printing were pre-crosslinked by mixing with $90 \mathrm{mM} \mathrm{CaCl}_{2}$ (final concentration of $18 \mathrm{mM} \mathrm{CaCl}_{2}$ ). ${ }^{[51]}$

\section{Rheological assessment of cECM-functionalized bioinks}

To assess if the bioinks are shear-thinning, their rheological properties were assessed using a MCR 301 rheometer (Anton Paar $\mathrm{GmbH}$, Austria). Bioinks before and after $\mathrm{CaCl}_{2}$ mediated pre-crosslinking were tested to assess how the viscosity varies with the pre-crosslinking, and to assess if the presence 
and concentration of $\mathrm{CECM}$ alters the viscosity of the bioinks. About $1 \mathrm{~mL}$ of each bioink was placed between $50 \mathrm{~mm}$ parallel plates with a measurement gap of $0.55 \mathrm{~mm}$. To determine the linear viscoelastic range of the bioinks, a viscosity ramp test was performed, where the viscosity of the bioinks was measured in the range of $0.11 / \mathrm{s}$ to $10001 / \mathrm{s}$ at the frequency of $1 \mathrm{~Hz}$. In addition to shear-thinning property of the bioinks, it is critical for the bioinks to be thixotropic, a rheological property that shows how quickly the bioink can recover its viscosity immediately after a high shear rate is applied for a short period of time (mimicking the 3D-printing process) and thus display structural integrity. Thus, a viscosity recovery test was carried out where the bioinks were subjected to $11 / \mathrm{s}$ shear rate for $60 \mathrm{~s}, 1001 / \mathrm{s}$ for $10 \mathrm{~s}$ and 1 1/s for $60 \mathrm{~s}$ to simulate the printing process, and to assess the recovery of the viscosity of the materials. The 100 1/s shear rate is estimated to be the maximum shear rate experienced by the hydrogel during printing process in general. ${ }^{[57]}$

\section{D-printing and culture of cECM-functionalized bioinks}

Cylindrical constructs of the bioinks encapsulated with MSCs were fabricated by layer-by-layer deposition using the 3D Discovery multi-head bioprinting system (Regen HU, Switzerland) operated within a laminar flow hood to ensure sterility throughout the fabrication process. The pre-crosslinked bioink was loaded into the pressure driven piston system and deposited on a flat surface using a pressure of $0.1 \mathrm{MPa}, 2-8 \mathrm{~mm} / \mathrm{s}$ piston speed and a $23 \mathrm{G}$ needle. As the printing parameters and the rheology of the bioinks impact cell viability and behavior, ${ }^{[63]}$ the aforementioned parameters were chosen to minimize the shear stresses experienced by the cells (measured via cell viability assays). The filament-spreading ratio, defined as the width of the printed filament divided by the needle diameter, was used as a measure of printability as previously described. ${ }^{[51]} 3 \mathrm{D}$-printing of the constructs was performed using an orthogonal 90-degree angle print round pattern for each layer, to obtain a cylindrical construct with a build height of $3 \mathrm{~mm}$ and a diameter of $5 \mathrm{~mm}$. The printed constructs were then ionically cross-linked using $60 \mathrm{mM} \mathrm{CaCl}_{2}$ for 20 min post-printing and cultured at $37^{\circ} \mathrm{C}$ for 21 or 42 days at 37 ${ }^{\circ} \mathrm{C}, 5 \% \mathrm{CO}_{2}$, and $5 \% \mathrm{O}_{2}$ (low oxygen conditions are known to be more conducive to chondrogenic differentiation) ${ }^{[64]}$ in FCDM. The media was changed twice a week. 
For TGF- $\beta 3$ release study, the bioinks were loaded with TGF- $\beta 3$ as opposed to adding it into the FCDM. In this case, each construct was cultured in $2.5 \mathrm{~mL}$ of FCDM (without TGF- $\beta 3$ ) and was changed twice a week. The bioinks were also prepared as previously described using a dual-syringe approach, and were encapsulated with the equivalent amount of TGF- $\beta 3$ as added to the media over the course of a 3 week study $(150 \mathrm{ng})$, along with pBMSCs $\left(20 \times 10^{6}\right.$ cells $\left./ \mathrm{mL}\right)$ before finally pre-crosslinking with $\mathrm{CaCl}_{2}$ for 3D-printing. ${ }^{[25]}$ An alginate only bioink with TGF- $\beta 3$ supplemented in the FCDM was used as a control. Constructs were then cultured at $37^{\circ} \mathrm{C}, 5 \% \mathrm{CO}_{2}$, and $5 \% \mathrm{O}_{2}$ for 21 days. Media was changed twice a week with FCDM (without TGF- $\beta 3$ ) and a portion of the changed media was collected for assessing the release profile of TGF- $\beta 3$ from the constructs.

\section{D-printing of polycaprolactone (PCL) framework and fabrication of hybrid construct.}

For polycaprolactone (PCL) $(\mathrm{Mn}=47,500$, Perstorp, United Kingdom) printing, fused deposition modelling (3D Discovery, Regen HU, Switzerland) was used to deposit filaments of molten PCL via a $30 \mathrm{G}(0.159 \mu \mathrm{m})$ needle. The printing parameters were extensively optimized in terms of needle size, printing speed, melting temperature of PCL, fiber spacing, alignment and pattern to fabricate a PCL frame that had a compressive modulus of $0.33 \mathrm{MPa}$, comparable to that of native $\mathrm{AC}$. ${ }^{[31]}$ Using these parameters, a highly porous 3D-printed PCL frame was printed with an orthogonal architecture including offset layers and a filament spacing of $1.5 \mathrm{~mm}$. The optimal offset was found to be half the filament spacing. The entire 3D-printed frame had the dimensions of $9 \mathrm{~mm} \times 9 \mathrm{~mm} \times 4 \mathrm{~mm}$. To characterize and confirm the filament size and spacing between fibers post printing, PCL scaffolds were imaged using SEM (Carl Zeiss, Germany) (data not shown). To fabricate the hybrid construct, the 3D-printed PCL frameworks were first ETO sterilized, and then, the empty pores were filled with the pre-crosslinked selected bioinks in sterile conditions using a $23 \mathrm{G}$ needle. The hybrid constructs were then crosslinked in $60 \mathrm{mM} \mathrm{CaCl}_{2}$ for $20 \mathrm{~min}$ and cultured in FCDM for up to 21 days at $37^{\circ} \mathrm{C}, 5 \% \mathrm{CO}_{2}$, and $5 \% \mathrm{O}_{2}$. 


\section{Cell viability assessment of 3D-printed constructs}

A two-color fluorescence assay was used to assess the cell viability within 24 hours of 3D-printing, 21 and, or 42 days of culture as previously described. ${ }^{[43]}$ Leica SP8 Confocal Microscope at $488 \mathrm{~nm}$ and $543 \mathrm{~nm}$ wavelengths was used for imaging and the viability was quantified using Image $\mathrm{J}$ software.

\section{Biochemical Assays}

Constructs were evaluated for their DNA, glycosaminoglycan (GAG), and total collagen content at day 0 (at the start of the experiment), 21 and 42 days of culture using previously established protocols. ${ }^{[43-44,}$ ${ }^{51]}$ For study using hBMSCs, calcium content was also assessed.[64] Four constructs per group were analyzed. The biochemical synthesis of each bioink was normalized to its own DNA content. Net collagen synthesis/DNA was calculated as \{total collagen production (at day 21 or 42 ) - total residual collagen (at day 0)\}/DNA at day 21 or 42 respectively. This accounts for any collagen present in the bioinks, especially in the cECM-functionalized bioinks at the start of the experiment. The same normalization was performed for determining net GAG/DNA and calcium/DNA at the end of day 21 or day 42.

\section{Histological assessment}

Histological assessment was carried out at three-time points: day 0 (at the start of the experiment), day 21 and day 42. Day 0 samples shows the background staining of the bioink if any, and serves as a reference control. Constructs were fixed in $4 \%$ paraformaldehyde suitable for alginate-based bioinks before being dehydrated in a series of ethanol baths of increasing concentrations and xylene, embedded in paraffin wax, sectioned at $7 \mu \mathrm{m}$ with a microtome (Leica RM2125RT) and affixed to microscope slides. Prior to staining, sections were deparaffinized and rehydrated in a series of xylene followed by alcohol baths of decreasing concentrations. The deposition of sGAG was determined using Alcian-blue and Aldehyde Fuchsin staining, whereas, collagen and calcium depositions were evaluated using Picrosirius Red and Alizarin Red staining respectively. ${ }^{[51]}$ All reagents were purchased from Sigma-Aldrich, Ireland. At protein level, collagen types I, II and X were evaluated using a standard immunohistochemical 
techniques. ${ }^{[39]}$ For positive and negative controls: ligament and $A C$ for collagen type I; $A C$ and ligament for collagen type II; and growth plate and ligament for collagen type $\mathrm{X}$ were used respectively. The semi-quantitative scoring of the staining was also performed using Image $\mathrm{J}$ using an established protocol in the literature. ${ }^{[65]}$

\section{ELISA assay and assessment of TGF- $\beta 3$ release profile}

During the TGF- $\beta 3$ release experiment, the supernatant media was collected at each medium change and the amount of TGF- $\beta 3$ released into the medium was quantified. ${ }^{[25,62]}$ Enzyme-Linked ImmunoSorbent Assay (ELISA) kit (DuoSet ${ }^{\circledR}$ ELISA DY243, Development System) following the manufacturer's protocol. TGF- $\beta 3$ content was deduced by calculating against a standard curve created using a four-parameter logistic (4-PL) curve-fit.

\section{Total RNA isolation, cDNA preparation, real-time qPCR and quantification}

To assess gene expression, RNA isolation, cDNA preparation and RT-PCR was performed following the previously established protocols. ${ }^{[44,58]}$ The KiCqStart $\circledast$ SYBR $\circledast$ Green Primers predesigned from Sigma Aldrich (Ireland) were used for all the genes. RT-PCR data was analyzed using Livak's delta delta CT method. For endogenous control and normalization, two housekeeping genes (GAPDH and 18S) were assessed and their geometric mean was used. ${ }^{[66]}$ However, only one housekeeping gene, 18S, was used in the case of pBMSCs. For normalization within the bioinks, alginate only bioink at day 21 was used as a reference.

\section{Mechanical testing of constructs before and after mechanical reinforcement}

Constructs were mechanically tested $(n=4)$ in unconfined compression using a standard material testing machine with either a $5 \mathrm{~N}$ and $100 \mathrm{~N}$ load cell (Zwick Roell Z005) for 3D-printed bioink constructs and PCL reinforced constructs respectively. ${ }^{\left[{ }^{39]}\right.}$ Briefly, constructs were kept hydrated through immersion in DMEM (Gibco Biosciences, Ireland) bath maintained at room temperature. The mechanical properties of all scaffold configurations ( $n=4$ per group) were assessed first, via ramp compression tests to $50 \%$ 
strain at a rate of $1 \mathrm{~mm} / \mathrm{min}$. Elastic moduli were calculated from the initial linear region of the obtained stress-strain curves.

\section{Statistical analysis}

All the data is expressed as mean \pm standard error unless otherwise stated. A minimum sample number of 4 were maintained throughout the study. For statistical assessment, normality of the data was tested and based on the normality of the data; either parametric (t-test or ANOVA followed by Tukey's post hoc test) or non-parametric (Mann-Whitney $U$ test or Kruskal-Wallis test) was used to determine the statistical significance. Graph Pad Prism 7.0 (GraphPad Software for Mac OS X, California) was used to perform all the statistical analysis.

\section{Results}

\section{Rheological properties and 3D-printability of cartilage ECM functionalized bioinks}

Porcine articular cartilage derived extracellular matrix (cECM) functionalized bioinks were developed using alginate as the base hydrogel. Briefly, hBMSCs (20 million cells $/ \mathrm{mL}$ ) were mixed with solubilized cECM and subsequently mixed with sodium alginate (2.45\% in DMEM) to make two bioinks (Fig. 1A): alginate $+0.2 \%(\mathrm{w} / \mathrm{v}) \mathrm{cECM}$ and alginate $+0.4 \% \mathrm{cECM}$. An alginate only bioink containing hBMSCs was used as a control. To characterize the printability of these bioinks, their rheological properties were first assessed. First, the viscosity of the bioinks (with and without $\mathrm{CaCl}_{2}$ mediated pre-crosslinking) was measured as a function of shear rate (Figure 1B). As expected, the viscosity of the hydrogel bioinks dramatically increased with pre-crosslinking. The pre-crosslinked bioinks were also shear-thinning. Functionalization with cECM had little effect on the viscosity of the bioinks. 
A)

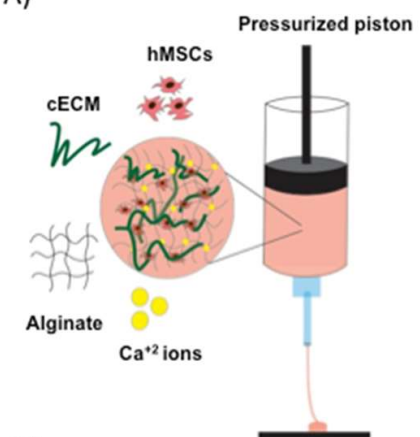

D)

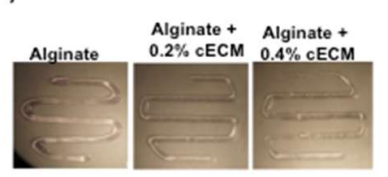

3D-printed cylindrical constructs (i) 80
B)
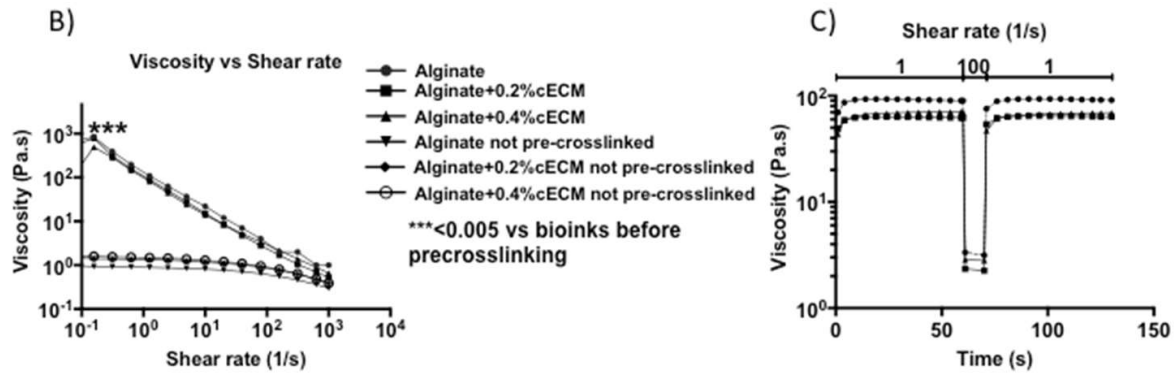

E)

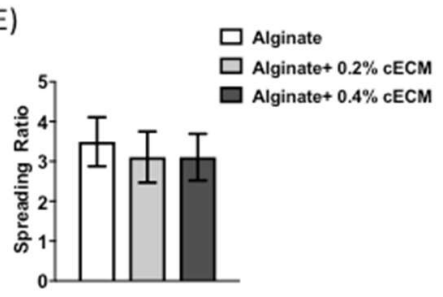

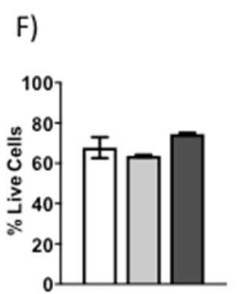

Figure 1. 3D-printability and rheological properties of CECM-functionalised bioinks encapsulated with hBMSCs. A) Schematic of the 3D-printing process using cECM-functionalized bioinks. B) Viscosity of the bioinks (with and without pre-crosslinking) in the presence of shear rate in the range of 0.1 to 1000 1/s at a frequency of $1 \mathrm{~Hz}$. C) Viscosity recovery test of the pre-crosslinked bioinks subjected to a shear rate increasing from 1 to 100 1/s for $10 \mathrm{~s}$, mimicking the 3D-printing process. D-E) Bioinks 3Dprinted in a defined pattern with structural integrity measured as the filament spreading ratio (width of the printed filament divided by the needle diameter) and quantified using ImageJ. Also shown are the representative 3D-printed cylindrical constructs. F) Quantification of cell viability in 3D-printed bioinks after 24 hours of culture. G) Cell viability within the 3D-printed bioinks at various time points (day 1, 21 and 42) throughout the culture duration. Green and red indicate live and dead cells respectively. $N=4$; Data expressed as mean \pm SE.

Next, a viscosity recovery test on the pre-crosslinked bioinks was performed in the presence of a steady shear rate to determine if the bioinks are thixotropic. Briefly, the pre-crosslinked bioinks were subjected to a $11 / \mathrm{s}$ shear rate for $60 \mathrm{~s}$, a $1001 / \mathrm{s}$ shear rate for $10 \mathrm{~s}$ and a $11 / \mathrm{s}$ shear rate for $60 \mathrm{~s}$ to simulate the printing process. The 100 1/s shear rate is estimated to be the maximum shear rate experienced by 
the bioinks during printing process. ${ }^{[57]}$ The viscosity of all the bioinks dropped by more than 10 -fold once the shear rate changed from 1 to 100 1/s (Figure 1C). The viscosity of all the bioinks also recovered almost immediately (within a few seconds), once the shear rate was reduced from 100 to $11 / \mathrm{s}$, thus exhibiting thixotropic behavior.

The hBMSCs laden pre-crosslinked bioinks were then tested for 3D-printability, where filaments were deposited onto a glass slide using a $23 \mathrm{G}$ needle $(337 \mu \mathrm{m})$ and the filament-spreading ratio was measured (ratio of width of the printed filament to the needle diameter) (Figure 1D and 1E). The spreading ratios of three bioinks were similar and consistent (mean of 3.11 to 3.49 ) as evidenced by a low standard error. Moreover, the presence of cECM does not negatively impact the printing process. Consistent with the rheology data, bioinks not pre-crosslinked with $\mathrm{CaCl}_{2}$ were unprintable (data not shown). To investigate the effect of 3D-printing on cell-viability, the cell-laden bioinks were 3D-printed under sterile conditions. Approximately $70 \%$ of the cells were viable 24 hours after 3D-printing (Figure 1F). 3D-printed cell-laden cylinders were also cultured in chondrogenic medium for up to 42 days, with cell viability maintained throughout the culture period (Figure 1G).

\section{cECM-functionalized bioinks enhanced chondrogenesis of hBMSCs}

To assess the chondro-inductivity of the bioinks, the 3D-printed MSC laden constructs were cultured statically in chondrogenic media for up to 42 days in low oxygen conditions $\left(5 \% \mathrm{O}_{2}\right)$ known to support chondrogenesis. ${ }^{[64]}$ All RT-PCR data was normalized to the alginate only bioink at day 21 . At day 21 , the expression of SOX9 in bioinks functionalized with $0.4 \%$ cECM was comparable to the alginate only bioink, but significantly lower $(p<0.05)$ in the bioink functionalized with $0.2 \%$ cECM (Figure $2 A$ ). However, after day 42, SOX9 gene expression was similar in all the three bioinks. At day 21 , COLL II expression was similar in all three bioinks (Figure 2B), however, by day 42, expression was significantly higher in bioinks functionalized with $0.4 \% \mathrm{cECM}$. A similar $\mathrm{CECM}$ concentration dependent increase was also observed for ACAN gene expression (Figure 2C), with significantly higher expression observed 
in the $0.4 \%$ cECM-functionalized bioinks at day 21 and 42 . Similar cECM mediated increases in COLL I expression was also observed (Figure 2D).

A)

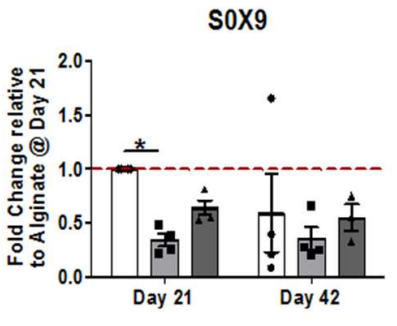

D)

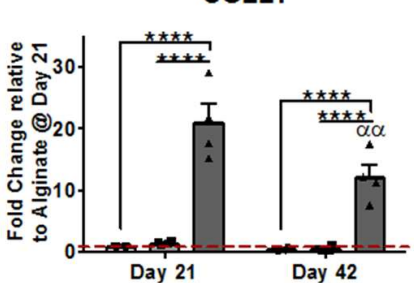

B)

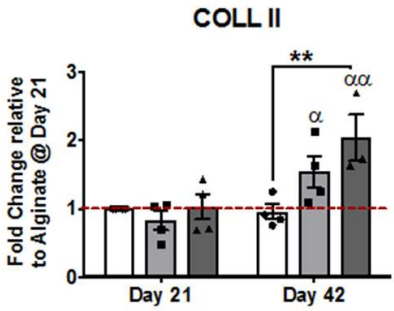

E)

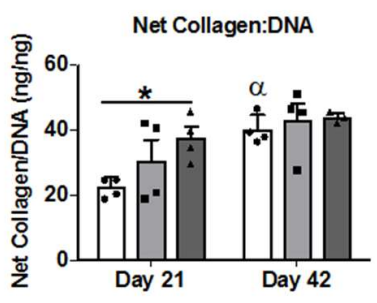

C)

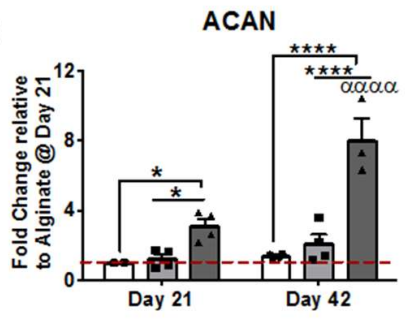

F)

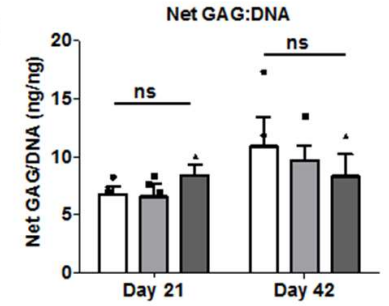

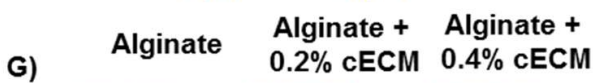

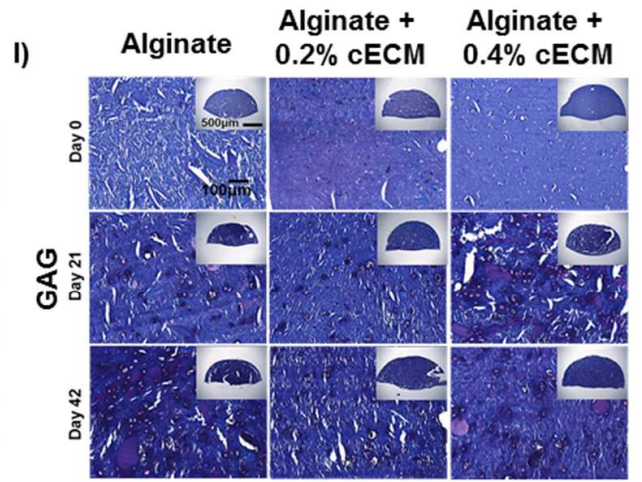

$\square$ Alginate

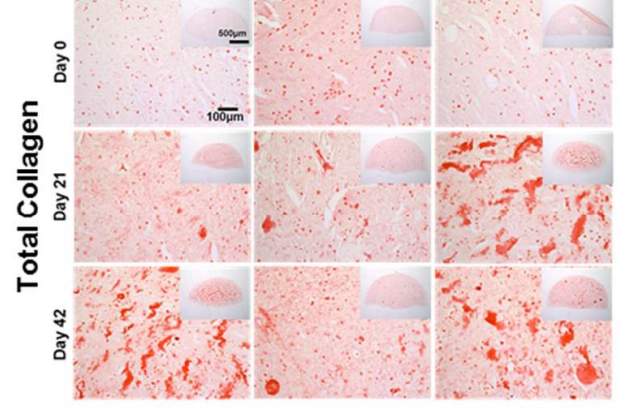

$\square$ Alginate+ $0.2 \%$ cECM $\square$ Alginate $0.4 \% \mathrm{cECM}$

$* * * *<0.0001, * *<0.01$ $<0.05$. $\alpha \alpha \alpha \alpha<0.0001, \alpha \alpha<0.01$ $a<0.05$ vs day 21 of the same bioink. $\mathrm{n}=4$ except for Alg $+0.4 \% \mathrm{cECM}$ @Day $42 \mathrm{n}=3$.
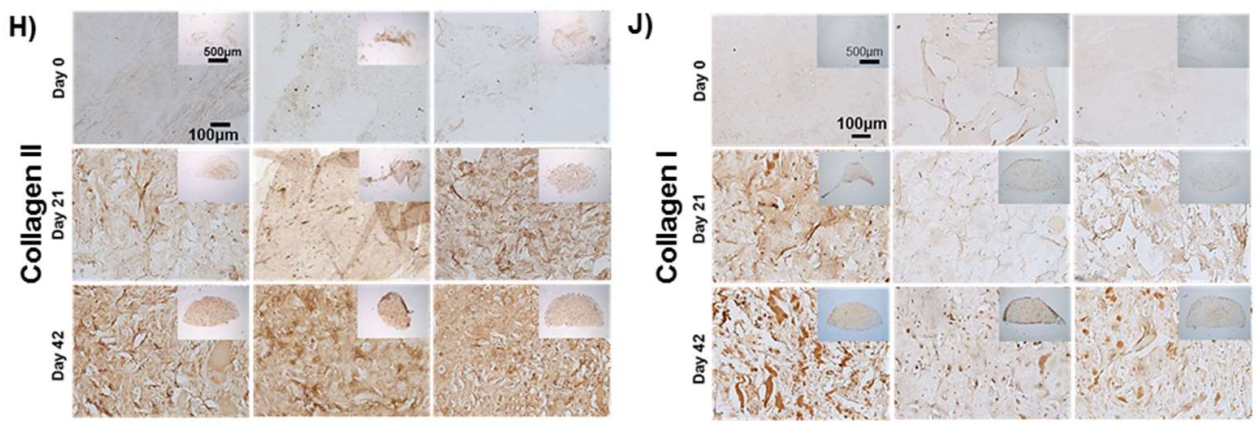

Figure 2. Enhanced chondrogenesis in cECM-functionalized bioinks. A-D) Expression of chondrogenic genes: SOX9, COLL II, ACAN and COLL I quantified using RT-PCR and analyzed using delta delta CT method, and normalized by respective gene expression in alginate only bioinks at day 21. E-F) Net collagen/DNA and GAG/DNA synthesis within the bioinks at day 21 and 42 . G-J) 
Histological and immunostaining bioinks at day 0,21 and 42: Picrosirius red staining showing total collagen production $(\mathrm{G})$, Coll II immunostaining $(\mathrm{H})$, Alcian-blue and Aldehyde Fuchsin staining for sGAG (I) and finally Coll I immunostaining $(\mathrm{J})$. $\mathrm{N}=4$. Data expressed as mean $\pm \mathrm{SE}$.

At day 21 , net collagen/DNA synthesis was significantly higher in bioinks functionalized with $0.4 \% \mathrm{cECM}$ (Figure 2E). More intense picrosirius red staining (Figure 2G) and Coll II immunostaining (Figure 2H) was also observed in the cECM-functionalized bioinks compared to day 0 (confirmed by semiquantification analysis of Coll II immunostaining intensity, see Supplementary Figure S1A). No significant differences in total collagen synthesis were observed at day 42. Although increases in COLL I gene expression was observed in $0.4 \%$ cECM bioink, the intensity of Coll I immunostaining was not noticeably higher in both the cECM-functionalized bioinks (Figure 2J, Supplementary Figure S1B). In fact, the Coll I immunostaining intensity was significantly higher in the alginate only bioink. Net sGAG/DNA synthesis and Alcian-blue and Aldehyde-Fuchsin staining (Figure $2 \mathrm{~F}$ and $2 \mathrm{l}$ ) was comparable in all three bioinks.

To evaluate if these responses are species-specific, and with a view towards a pre-clinical study in a swine model, the same experiments were repeated with the pBMSCs (supplementary figure S2). Interestingly, similar responses were observed with significant increases in SOX9, COLL II and ACAN gene expression and net collagen and GAG synthesis in CECM-functionalized bioinks. Another result from this study was the observation that functionalization of the bioinks with cECM alone is not sufficient to promote robust chondrogenic differentiation of the MSCs, as limited chondrogenesis was observed in constructs cultured in serum supplemented media compared to those stimulated with TGF- $\beta 3$ (Supplementary figure S2). Further, characteristic lacunae, increased cell number and a round cell morphology was only observed in constructs cultured in the presence of TGF- $\beta 3$. Thus, growth factor stimulation is essential for robust chondrogenesis of MSCs, even in the CECM-functionalized bioinks. 
cECM-functionalized bioinks promote the development of an endochondral-like phenotype

A major challenge in cartilage tissue engineering is that conditions that support robust chondrogenesis of MSCs also tend to promote the expression of genes associated with hypertrophy, endochondral bone formation and osteogenesis. ${ }^{[67-68]}$ Thus, the expression of RUNX2 and COLL $X$ in different bioinks was also investigated (Figure 3). Functionalization with $0.4 \%$ cECM increased the expression of RunX2 at both day 21 and 42 (Figure 3A), with significantly higher levels of calcium accumulation also observed in cECM-functionalized bioinks by day 42 (Figure 3C). Alizarin Red staining confirmed the presence of calcific deposits in cECM-functionalized bioinks at day 42 (Figure 3D). Interestingly, no significant differences in COLL $X$ gene expression (Figure 3B) or immunostaining (Figure 3E) were observed between the groups (also confirmed in supplementary figure S1C). Similar increases in the gene expression of RUNX2 and COLL $X$ with $\mathrm{CECM}$-functionalization were observed with pBMSCs (see supplementary figure S3).

A)

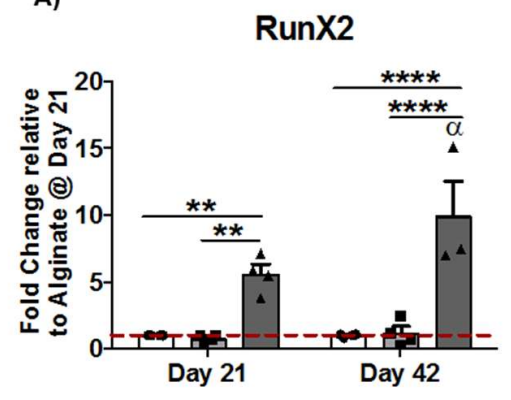

B)
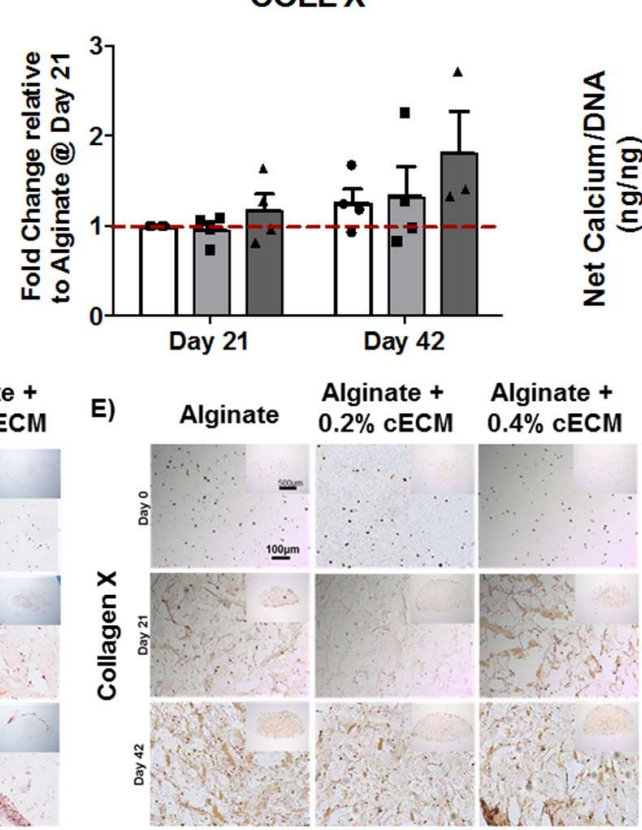

Net Calcium: DNA

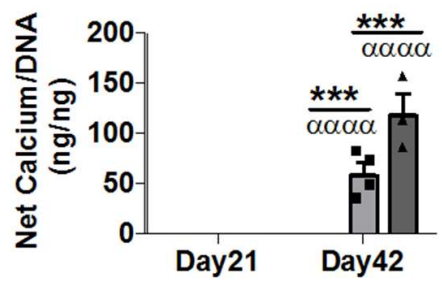

$\square$ Alginate

$\square$ Alginate+ $0.2 \% \mathrm{cECM}$

Alginate+ $0.4 \% \mathrm{cECM}$

$* * * *<0.0001,{ }^{* * * *}<0.001$, $* *<0.01$. $\alpha \alpha \alpha \alpha<0.0001, \alpha<0.05$ vs day 21 of the same bioink. $n=4$ except for Alg+0.4\% cECM @Day $42 n=3$.

Figure 3. $c E C M$-functionalized bioinks increase the expression of osteogenic or hypertrophic markers. A-B) Expression of RunX2 and COLL X, quantified using RT-PCR and normalized by respective gene expression in alginate only bioinks at day 21. C) Net calcium/DNA levels at day 21 and 
42, normalized by the DNA content of the respective bioinks. D-E) Histology and immunostaining at day 0, 21 and 42: Alizarin Red staining for mineralization (D) and immunostaining for Coll $X(E) . N=4$. Data expressed as mean $\pm \mathrm{SE}$.

\section{Release of TGF $\beta 3$ from cECM-functionalized bioinks promotes chondrogenesis of $p B M S C s$}

As growth factors such as TGF- $\beta 3$ are critical for promoting robust chondrogenesis of pBMSCs, controlled delivery of growth factors from bioprinted constructs may be critical to direct cartilage regeneration in vivo. With this in mind, we next assessed the capacity of cECM-functionalized bioinks to support the controlled delivery of TGF- $\beta 3$ (Figure 4A), and compared chondrogenesis in these growth factor eluting bioinks to that in control alginate bioinks where TGF- $\beta 3$ was directly supplemented into the media. 
A)
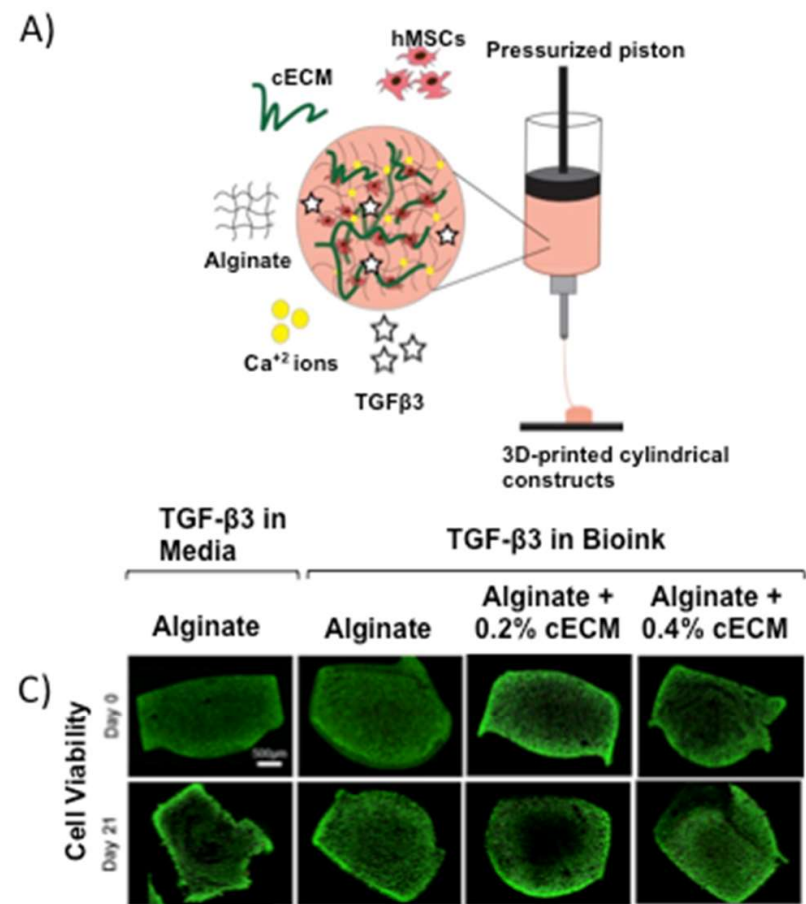

D)
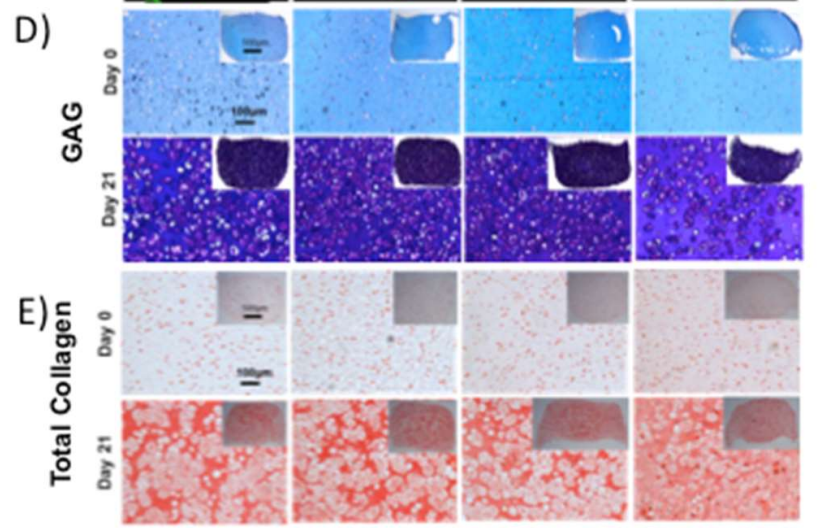

B)
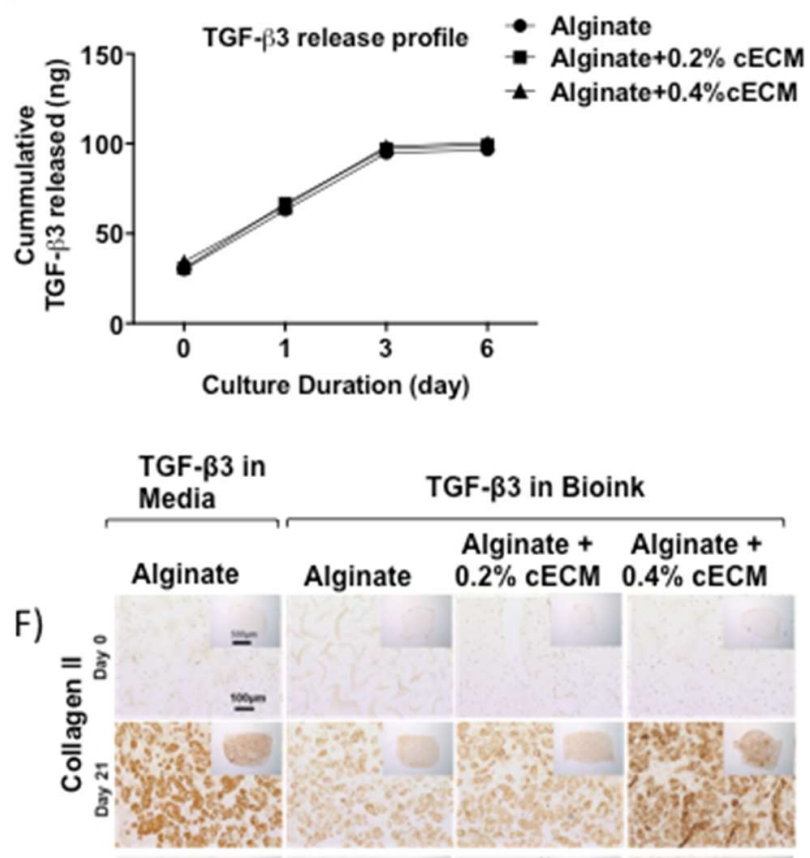

G)

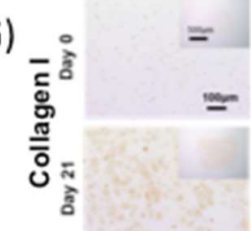

H)

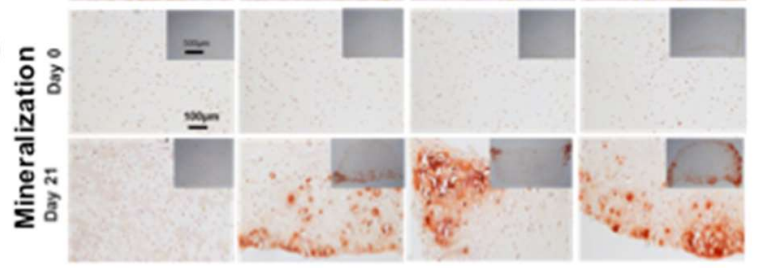

Figure 4. TGFß3 delivery from $\mathrm{CECM}$-functionalized bioinks promotes chondrogenesis of pBMSCs. A) Schematic of 3D-printing process of bioinks encapsulated with pBMSCs and TGF 33 . B) TGF $\beta 3$ release profile from the bioinks within first 6 days of 21 day culture assessed using an ELISA assay. C) Cell viability in bioinks throughout the culture period (day 0 and 21 ). Green and red indicate live and dead cells respectively. D-H) Histology and immunostaining at day 0 and 21: Alcian-blue and Aldehyde Fuchsin staining for GAG accumulation (D). Picrosirius red staining for total collagen production (E). Immunostaining for Coll II (F) and Coll I (G). Alizarin Red staining for mineralization $(H)$. $\mathrm{N}=4$. Data expressed as mean $\pm \mathrm{SE}$. 
A burst release of TGF- $\beta 3$ was observed for all three bioinks, with almost $66 \%$ of the TGF $\beta 3$ released within 1 week (Figure 4B). Good cell viability was also observed in all bioinks (Figure 4C). Alcian-blue and Aldehyde Fuchsin staining for sGAG (Figure 4D), and Picrosirius Red staining for collagen (Figure 4E), was comparable in all bioinks. Coll II immunostaining was more intense in $0.4 \%$ cECMfunctionalized bioink compared to the alginate only bioink (Figure 4F), comparable to that observed in the control bioink where TGF- $\beta 3$ was directly supplemented into the media. There was no appreciable staining for Coll I in any of the bioinks (Figure 4G). However, the intensity of Alizarin Red staining (for calcium deposition) was more intense in bioinks releasing TGF 33 compared to the control where TGF$\beta 3$ was directly supplemented into the media.

\section{Reinforcing cECM-functionalized bioinks with networks of 3D-printed PCL}

Having confirmed the capacity of cECM (0.4\%) functionalized bioinks for supporting robust chondrogenesis; we proceeded to reinforce this bioinks with networks of 3D-printed PCL for use in loadbearing applications. We achieved this by first 3D-printing a porous PCL framework (9x9x4mm), resulting in a scaffold with a compressive modulus of $0.33 \mathrm{MPa}$, similar to that of native AC (Figure 5C). ${ }^{[69]}$ Using a $23 \mathrm{G}$ needle, either alginate only bioink or $0.4 \% \mathrm{cECM}$-functionalized alginate bioink was extruded in the pores of the PCL framework (Figure 5A and $\mathrm{B}$ ). As expected, mechanical reinforcement of the bioinks with PCL dramatically improved the mechanical properties of the resulting construct (Figure 5C). After $24 \mathrm{hr}$ of culture, cells were homogeneously distributed within the bioinks throughout the PCL framework (Figure 5D), with longer-term studies confirming that the cells remained viable over 21 days of culture (Supplementary Figure S4). 
A)

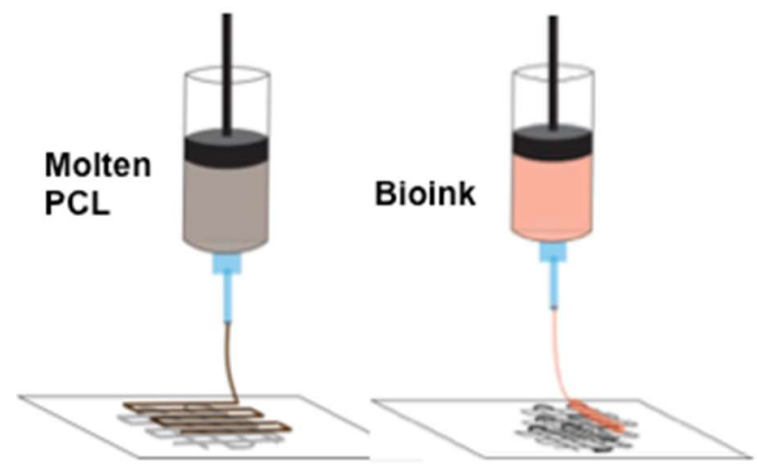

C)

Mechanical reinforcement of bioinks with a 3D printed PCL framework

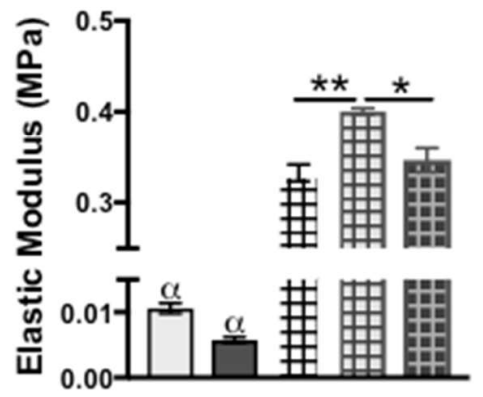

B)

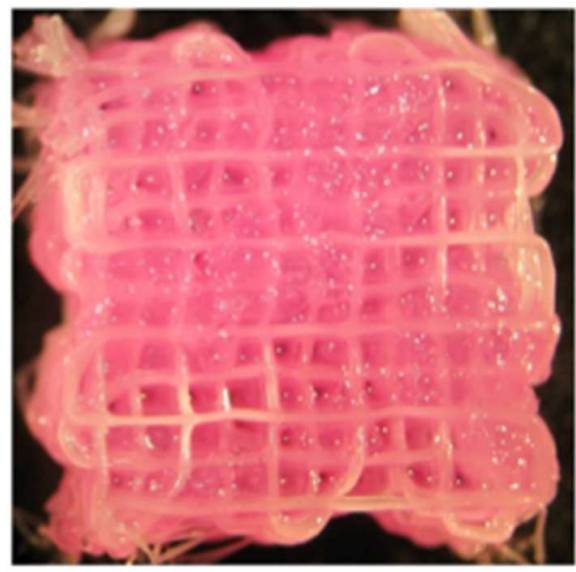

D) Cell viability after $24 \mathrm{hr}$ culture Alginate

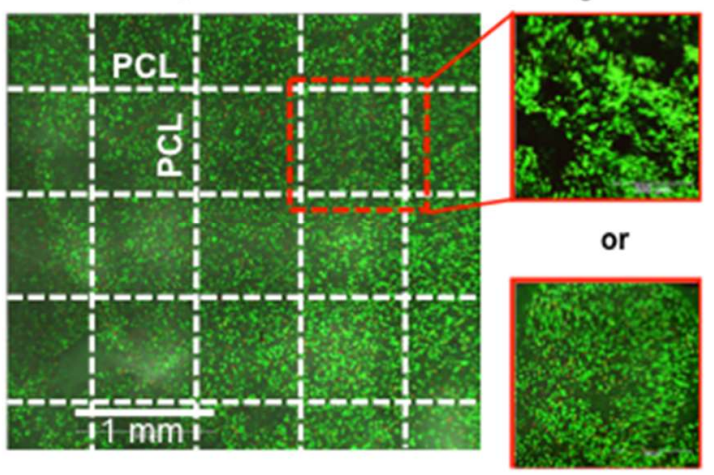

Alginate + $0.4 \% \mathrm{cECM}$

Figure 5. Reinforcing CECM-functionalized bioinks with networks of 3D-printed PCL. A) Schematic of 3D-printing process, consisting of fused deposition of PCL framework followed by infilling with bioink. B) Representative image of the hybrid construct after 3D-printing process. C) Comparison of elastic modulus of the bioinks, PCL frame only and hybrid construct. D) Representative images of hybrid construct showing cell viability of MSCs encapsulated in either alginate or $0.4 \% \mathrm{cECM}$ functionalized alginate bioink within the PCL framework after 24 hours of culture. Green and red indicate live and dead cells respectively. $\mathrm{N}=4$. Data expressed as mean $\pm \mathrm{SE}$. 


\section{Discussion}

Herein we described the development of cECM-functionalized bioinks and evaluated their rheological properties and suitability for 3D-bioprinting of cartilage-like tissues. These cECM-functionalized bioinks were found to enhance chondrogenesis of hBMSCs in a cECM concentration dependent manner. Furthermore, when these cECM-functionalized bioinks were used to deliver TGF- $\beta 3$, they were found capable of promoting robust chondrogenesis of MSCs. One concern was that $\mathrm{CECM}$ functionalization also enhanced the expression of markers often associated with hypertrophy and MSC osteogenesis or progression along an endochondral-like pathway. Finally, a multiple-tool fabrication system was successfully employed to demonstrate the feasibility of fabricating biomimetic cartilage-like implants using cECM-functionalized bioinks mechanically reinforced with a PCL framework.

Understanding the rheological properties (shear thinning and thixotropy) of bioinks is crucial for successful 3D-bioprinting (or injectability) and to ensure that the bioinks are capable of protecting the encapsulated cells. During the 3D-printing process, cells within the bioinks can experience high mechanical stresses that can lead to cell death. When pre-crosslinked with $\mathrm{CaCl}_{2}$, all the bioinks (with and without the $\mathrm{CECM}$ ) had significantly higher viscosity compared to their un-crosslinked counterparts and showed excellent shear-thinning and thixotropic behavior that can dramatically reduce shear induced cell damage during the printing process. ${ }^{[63]}$ Based on the printing parameters, the average shear rate experienced by the bioinks was estimated to be $1001 / \mathrm{s},{ }^{[57]}$ lower than other studies reported in the literature. ${ }^{[63]}$ Furthermore, a low piston pressure of $0.1 \mathrm{MPa}$ (1 bar) ensured a relatively low pressure drop was experienced by the cells. Thus, the 3D-printing parameters ensured that encapsulated cells did not experience excessive forces, resulting in high cell viability $(>70 \%)$ in all bioinks 24 hours after printing. The 3D-printed constructs also maintained their structural integrity over 42 days of culture.

The $0.4 \%$ cECM-functionalized bioink was found to be more chondro-inductive, as evident by increased gene expression of COLL II and ACAN, as well as increased synthesis of collagen. Although COLL I 
gene expression was also significantly higher with cECM-functionalization, this did not appear to translate into increased type I collagen protein production. The synthesis of sGAG was comparable among all the bioinks, indicating that the main benefit of $\mathrm{CECM}$ functionalization was increased collagen type II production. However, cECM-functionalized bioinks were also found to increase the expression of RUNX2 and promote higher levels of calcium deposition, suggesting that a sub-population of MSCs were undergoing osteogenesis in these bioinks, and/or that the chondrogenically primed MSCs were progressing along an endochondral-like pathway. This is in agreement with previous studies, which demonstrated that cartilage ECM derived biomaterials can promote hypertrophy ${ }^{[70-71]}$ and an endochondral-like phenotype. ${ }^{[32,}$ 70] Further studies are required to assess whether cECMfunctionalization will lead to endochondral bone formation in vivo if bioprinted constructs are implanted into cartilage defects, where mechanical loading of the joint may suppress hypertrophy and endochondral ossification ${ }^{[72-73]}$ of the bioprinted cartilage. If not, the utility of cECM functionalization may also lie in the bioprinting of constructs to promote large bone defect regeneration via an endochondral pathway. ${ }^{[32,74]}$

Functionalization of bioinks with $\mathrm{CECM}$ alone proved to be insufficient to induce robust chondrogenic differentiation of MSCs. As such, stimulation with growth factors such as TGF- $\beta 3$ to MSCs will likely be important to successful chondrogenic differentiation. ${ }^{[75]}$ Therefore for applications that envisage direct implantation of bioprinted constructs without any pre-culture, bioinks should also be capable of delivering growth factors to printed cells in a controlled fashion. ECM components such as collagen and proteoglycans can bind growth factors, ${ }^{[62,76]}$ however despite this, the release profile of TGF- $\beta 3$ was similar among the control and cECM-functionalized bioinks. This may be due to the relatively low concentrations of $\mathrm{CECM}$ with the alginate based bioinks. In spite of this, the $0.4 \% \mathrm{cECM}$-functionalized bioinks still supported robust chondrogenesis of MSCs, but was again accompanied by calcification of the engineered tissue, which was not observed in control bioinks where TGF- $\beta 3$ was directly supplemented into the media. The initial burst release of TGF- $\beta 3$ and the unavailability of sufficient

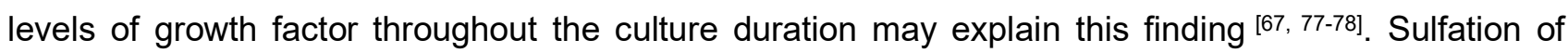


alginate can dramatically improve its capacity to bind to TGF- $\beta 3^{[79]}$ and can be incorporated into these bioinks for a more controlled and sustained TGF- $\beta 3$ release profile.

The compressive stiffness of the bioinks was found to be at least an order of magnitude lower than that of native articular cartilage. To address this concern, we reinforced the cECM bioinks with networks of 3D-printed PCL. PCL is widely used in 3D-printing owing to its mechanical strength, biocompatibility, low melting temperatures and thermal stability. $[39,46-48,74,80]$ 3D-printing parameters of PCL were optimized to fabricate a supporting framework with 1) sufficient porosity to allow uniform tissue ingrowth, and 2) a compressive modulus comparable to that of articular cartilage. The bioinks (with and without cECM-functionalization) were successfully reinforced with this 3D-printed PCL framework, resulting in a hybrid construct with biomimetic mechanical properties. Importantly, no noticeable loss in cell viability was observed with PCL reinforcement.

\section{Conclusions}

In summary, this study demonstrated the benefits of functionalizing alginate bioinks with cECM to enhance chondrogenesis of encapsulated MSCs. Functionalization of alginate with relatively small amounts of solubilized cECM significantly improved its chondro-inductive potential and promoted robust chondrogenesis of the MSCs, but with evidence to suggest that these cells continued to progress along an endochondral-like pathway. By depositing cECM-functionalized bioinks into networks of 3D-printed PCL, it was also possible to engineer biomimetic constructs with compressive mechanical properties in the same range as that of native cartilage. Thus, the bioinks developed in this study comprise a novel class of biomaterial for cell and/or growth factor delivery that can be used in the regeneration of musculoskeletal tissues.

\section{Acknowledgements}

Funding for this work was provided by European Research Council (JointPrint; ERC-CoG-2014-647004) and Science Foundation Ireland through the Investigators Programme (12/IA/1554). 


\section{References}

[1] J. Carnes, O. Stannus, F. Cicuttini, C. Ding, G. Jones, Osteoarthritis and Cartilage 2012, 20, 1541.

[2] M. R. Steinwachs, L. Engebretsen, R. H. Brophy, Cartilage 2011, 3, 11S.

[3] J. B. Driban, G. H. Lo, C. B. Eaton, L. L. Price, B. Lu, T. E. McAlindon, The Journal of Rheumatology 2015, 42, 1463.

[4] F. A. Khan, M. F. Koff, N. O. Noiseux, K. A. Bernhardt, M. M. O'Byrne, D. R. Larson, K. K. Amrami, K. R. Kaufman, Effect of Local Alignment on Compartmental Patterns of Knee Osteoarthritis, Vol. 90, 2008.

[5] T. L. Sanders, A. Pareek, M. R. Obey, N. R. Johnson, J. L. Carey, M. J. Stuart, A. J. Krych, The American Journal of Sports Medicine 2017, 45, 1799.

[6] C. Willers, D. J. Wood, M. H. Zheng, Journal of Musculoskeletal Research 2003, 07, 157.

[7] P. G. Bullough, E. F. DiCarlo, Annals of the Rheumatic Diseases 1990, 49, 412.

[8] D. C. Flanigan, J. D. Harris, T. Q. Trinh, R. A. Siston, R. H. Brophy, Med Sci Sports Exerc 2010, 42, 1795.

[9] A. J. Sophia Fox, A. Bedi, S. A. Rodeo, Sports Health 2009, 1, 461.

[10] A. Guermazi, D. Hayashi, F. W. Roemer, J. Niu, E. K. Quinn, M. D. Crema, M. C. Nevitt, J. Torner, C. E. Lewis, D. T. Felson, Arthritis \& rheumatology (Hoboken, N.J.) 2017, 69, 560.

[11] K. Rönn, N. Reischl, E. Gautier, M. Jacobi, Arthritis 2011, 2011, 9.

[12] P. Kane, R. Frederick, B. Tucker, C. C. Dodson, J. A. Anderson, M. G. Ciccotti, K. B.

Freedman, The Physician and Sportsmedicine 2013, 41, 75.

[13] C. Vinatier, J. Guicheux, Annals of Physical and Rehabilitation Medicine 2016, 59, 139.

[14] A. Goldberg, K. Mitchell, J. Soans, L. Kim, R. Zaidi, Journal of Orthopaedic Surgery and Research 2017, 12, 39.

[15] G. Filardo, F. Perdisa, A. Roffi, M. Marcacci, E. Kon, Journal of Orthopaedic Surgery and Research 2016, 11, 42. 
[16] M. Lo Monaco, G. Merckx, J. Ratajczak, P. Gervois, P. Hilkens, P. Clegg, A. Bronckaers, J.-M. Vandeweerd, I. Lambrichts, Stem Cells International 2018, 2018, 22.

[17] C. Karlsson, C. Brantsing, T. Svensson, H. Brisby, J. Asp, T. Tallheden, A. Lindahl, Journal of Orthopaedic Research 2007, 25, 152.

[18] M. Tamaddon, M. Burrows, S. A. Ferreira, F. Dazzi, J. F. Apperley, A. Bradshaw, D. D. Brand, J. Czernuszka, E. Gentleman, Scientific Reports 2017, 7, 43519.

[19] A. Matsiko, T. J. Levingstone, J. P. Gleeson, F. J. O'Brien, Advanced Healthcare Materials 2015, 4, 1175.

[20] M. Rutgers, D. B. Saris, L. A. Vonk, M. H. van Rijen, V. Akrum, D. Langeveld, A. van Boxtel, W. J. Dhert, L. B. Creemers, Tissue Engineering Part A 2012, 19, 59.

[21] J. S. Pieper, P. M. van der Kraan, T. Hafmans, J. Kamp, P. Buma, J. L. C. van Susante, W. B. van den Berg, J. H. Veerkamp, T. H. van Kuppevelt, Biomaterials 2002, 23, 3183.

[22] X. Yang, Z. Lu, H. Wu, W. Li, L. Zheng, J. Zhao, Materials Science and Engineering: C 2018, 83, 195.

[23] L. ZuFu, D. Behrouz Zandieh, H. ChunLing, B. Ruud A., H. Marco N., Tissue Engineering Part A 2010, 16, 81.

[24] L. Zheng, H. S. Fan, J. Sun, X. N. Chen, G. Wang, L. Zhang, Y. J. Fan, X. D. Zhang, Journal of Biomedical Materials Research Part A 2010, 93A, 783.

[25] H. V. Almeida, Y. Liu, G. M. Cunniffe, K. J. Mulhall, A. Matsiko, C. T. Buckley, F. J. O’Brien, D. J. Kelly, Acta Biomaterialia 2014, 10, 4400.

[26] K. Lindenhayn, C. Perka, R.-S. Spitzer, H.-H. Heilmann, K. Pommerening, J. Mennicke, M. Sittinger, Journal of Biomedical Materials Research 1999, 44, 149.

[27] E. Amann, P. Wolff, E. Breel, M. van Griensven, E. R. Balmayor, Acta Biomaterialia 2017, 52, 130.

[28] A. Matsiko, T. J. Levingstone, F. J. O'Brien, J. P. Gleeson, Journal of the Mechanical Behavior of Biomedical Materials 2012, 11, 41. 
Biomedical Materials Research Part B: Applied Biomaterials 2018, 0.

[30] T. Wang, F. Yang, Stem Cell Research \& Therapy 2017, 8, 284.

[31] F. Pati, J. Jang, D.-H. Ha, S. Won Kim, J.-W. Rhie, J.-H. Shim, D.-H. Kim, D.-W. Cho, Nature Communications 2014, 5, 3935.

[32] D. Gawlitta, K. E. M. Benders, J. Visser, A. S. van der Sar, D. H. R. Kempen, L. F. H. Theyse, J. Malda, W. J. A. Dhert, Tissue Engineering Part A 2015, 21, 694.

[33] E. C. Beck, M. Barragan, M. H. Tadros, S. H. Gehrke, M. S. Detamore, Acta biomaterialia 2016, 38, 94 .

[34] C. R. Rowland, K. A. Glass, A. R. Ettyreddy, C. C. Gloss, J. R. L. Matthews, N. P. T. Huynh, F. Guilak, Biomaterials 2018, 177, 161.

[35] C. R. Rowland, L. A. Colucci, F. Guilak, Biomaterials 2016, 91, 57.

[36] A. D. Dikina, H. V. Almeida, M. Cao, D. J. Kelly, E. Alsberg, ACS Biomaterials Science \& Engineering 2017, 3, 1426.

[37] L. Luo, J. Y. J. Chu, R. Eswaramoorthy, K. J. Mulhall, D. J. Kelly, ACS Biomaterials Science \& Engineering 2017, 3, 1933.

[38] L. Luo, R. Eswaramoorthy, K. J. Mulhall, D. J. Kelly, Journal of the Mechanical Behavior of Biomedical Materials 2016, 55, 21.

[39] A. C. Daly, G. M. Cunniffe, B. N. Sathy, O. Jeon, E. Alsberg, D. J. Kelly, Advanced Healthcare Materials 2016, 5, 2352.

[40] S. E. Critchley, D. J. Kelly, Journal of 3D Printing in Medicine 2017, 1, 269.

[41] T. Guo, J. Lembong, L. G. Zhang, J. P. Fisher, Tissue Engineering Part B: Reviews 2017, 23, 225.

[42] D. Nguyen, D. A. Hägg, A. Forsman, J. Ekholm, P. Nimkingratana, C. Brantsing, T.

Kalogeropoulos, S. Zaunz, S. Concaro, M. Brittberg, A. Lindahl, P. Gatenholm, A. Enejder, S. Simonsson, Scientific Reports 2017, 7, 658.

[43] F. E. Freeman, D. J. Kelly, Scientific Reports 2017, 7, 17042. 
S. Romanazzo, S. Vedicherla, C. J. Moran, D. J. Kelly, Meniscus ECM-functionalised hydrogels containing infrapatellar fat pad-derived stem cells for bioprinting of regionally defined meniscal tissue, 2017.

[45] A. C. Daly, F. E. Freeman, T. Gonzalez-Fernandez, S. E. Critchley, J. Nulty, D. J. Kelly, Advanced Healthcare Materials 2017, 6, 1700298.

[46] S. Martinez-Diaz, N. Garcia-Giralt, M. Lebourg, J.-A. Gómez-Tejedor, G. Vila, E. Caceres, P. Benito, M. Monleón Pradas, X. Nogues, J. L. Gómez Ribelles, J. C. Monllau, The American Journal of Sports Medicine 2010, 38, 509.

[47] E. Malikmammadov, T. E. Tanir, A. Kiziltay, V. Hasirci, N. Hasirci, Journal of Biomaterials Science, Polymer Edition 2018, 29, 863.

[48] A. D. Olubamiji, Z. Izadifar, J. L. Si, D. M. L. Cooper, B. F. Eames, D. X. B. Chen, Biofabrication 2016, 8, 025020.

[49] A. Salamon, S. van Vlierberghe, I. van Nieuwenhove, F. Baudisch, G.-J. Graulus, V. Benecke, K. Alberti, H.-G. Neumann, J. Rychly, J. C. Martins, P. Dubruel, K. Peters, Materials 2014, 7, 1342.

[50] G. Portocarrero Huang, R. Menezes, R. Vincent, W. Hammond, L. Rizio, G. Collins, T. L. Arinzeh, Tissue Engineering Part A 2017, 23, 1011.

[51] A. C. Daly, S. Critchley, E. M. Rencsok, D. J. Kelly, Biofabrication 2016, 8, 045002.

[52] K. E. M. Benders, P. R. v. Weeren, S. F. Badylak, D. B. F. Saris, W. J. A. Dhert, J. Malda, Trends in Biotechnology 2013, 31, 169.

[53] S. J. Bidarra, C. C. Barrias, P. L. Granja, Acta Biomaterialia 2014, 10, 1646.

[54] M. Szekalska, A. Pucilowska, E. Szymańska, P. Ciosek, K. Winnicka, International Journal of Polymer Science 2016, 2016, 17.

[55] J. A. Rowley, G. Madlambayan, D. J. Mooney, Biomaterials 1999, 20, 45.

[56] R. Olivares-Navarrete, E. M. Lee, K. Smith, S. L. Hyzy, M. Doroudi, J. K. Williams, K. Gall, B. D. Boyan, Z. Schwartz, PLoS ONE 2017, 12, e0170312.

[57] H. Li, S. Liu, L. Lin, International Journal of Bioprinting; Vol 2, No 2 (2016) 2016. 
[58] T. Gonzalez-Fernandez, E. G. Tierney, G. M. Cunniffe, F. J. O'Brien, D. J. Kelly, Tissue Engineering Part A 2016, 22, 776.

[59] S.-W. Kang, B.-H. Cha, H. Park, K.-S. Park, K. Y. Lee, S.-H. Lee, Macromolecular Bioscience 2011, 11, 673 .

[60] N. Iwasaki, S.-T. Yamane, T. Majima, Y. Kasahara, A. Minami, K. Harada, S. Nonaka, N. Maekawa, H. Tamura, S. Tokura, M. Shiono, K. Monde, S.-I. Nishimura, Biomacromolecules 2004, 5, 828.

[61] D. P. Lennon, A. I. Caplan, Experimental Hematology 2006, 34, 1604.

[62] H. V. Almeida, K. J. Mulhall, F. J. O'Brien, D. J. Kelly, Journal of Tissue Engineering and Regenerative Medicine 2017, 11, 2979.

[63] B. A. Aguado, W. Mulyasasmita, J. Su, K. J. Lampe, S. C. Heilshorn, Tissue Engineering. Part A 2012, 18, 806.

[64] E. J. Sheehy, C. T. Buckley, D. J. Kelly, Biochemical and Biophysical Research Communications 2012, 417, 305.

[65] F. Varghese, A. B. Bukhari, R. Malhotra, A. De, PLOS ONE 2014, 9, e96801.

[66] J. Vandesompele, K. De Preter, F. Pattyn, B. Poppe, N. Van Roy, A. De Paepe, F. Speleman, Genome Biology 2002, 3, research0034.1.

[67] M. B. Mueller, R. S. Tuan, Arthritis and rheumatism 2008, 58, 1377.

[68] N. P. T. Huynh, B. Zhang, F. Guilak, The FASEB Journal, 0, fj.201800534R.

[69] F. Boschetti, G. Pennati, F. Gervaso, G. M Peretti, G. Dubini, Biomechanical properties of human articular cartilage under compressive loads, Vol. 41, 2004.

[70] A. J. Sutherland, E. C. Beck, S. C. Dennis, G. L. Converse, R. A. Hopkins, C. J. Berkland, M. S. Detamore, PLOS ONE 2015, 10, e0121966.

[71] B. B. Rothrauff, G. Yang, R. S. Tuan, Stem Cell Research \& Therapy 2017, 8, 133.

[72] S. F. Carroll, C. T. Buckley, D. J. Kelly, Journal of Biomechanics 2014, 47, 2115.

[73] S. D. Thorpe, T. Nagel, S. F. Carroll, D. J. Kelly, PLOS ONE 2013, 8, e60764.

[74] A. C. Daly, P. Pitacco, J. Nulty, G. M. Cunniffe, D. J. Kelly, Biomaterials 2018, 162, 34. 
[75] A. Tekari, R. Luginbuehl, W. Hofstetter, R. J. Egli, PLoS ONE 2015, 10, e0120857.

[76] L. Macri, D. Silverstein, R. A. F. Clark, Advanced Drug Delivery Reviews 2007, 59, 1366.

[77] A. M. Mackay, S. C. Beck, J. M. Murphy, F. P. Barry, C. O. Chichester, M. F. Pittenger, Tissue Engineering 1998, 4, 415.

[78] K. Liu, G. D. Zhou, W. Liu, W. J. Zhang, L. Cui, X. Liu, T. Y. Liu, Y. Cao, Biomaterials 2008, 29, 2183.

[79] T. Re'em, Y. Kaminer-Israeli, E. Ruvinov, S. Cohen, Biomaterials 2012, 33, 751.

[80] E. J. Levorson, M. Santoro, F. K. Kasper, A. G. Mikos, Acta biomaterialia 2014, 10, 1824. 